\title{
COHOMOLOGY OF NONASSOCIATIVE ALGEBRAS
}

\author{
BY NEAL D. GLASSMAN
}

Communicated by G. D. Mostow, January 15, 1968

The theories of associative and Lie cohomology for finite dimensional algebras over fields have much in common. Let $a$ be an associative algebra with unit over the field $K, M$ a unital $a$-bimodule; let $\mathfrak{L}$ be a Lie algebra over $K, N$ an $\&$-bimodule. Define $H^{n}(a, M)$ $=\operatorname{Ext}^{n} Q_{\otimes_{K}} Q^{0}(Q, M), H^{n}(\mathscr{L}, N)=\operatorname{Ext}_{U(L)}^{n}(K, N)$. Here $Q^{0}$ is the opposite algebra of $\mathfrak{Q}, U(\mathfrak{L})$ is the universal enveloping algebra of $\mathscr{L}, \mathfrak{Q}$ is regarded as the regular $a$-bimodule, and $K$ is regarded as a trivial \&-bimodule. We find that

(i) $H^{1}(\propto, M), H^{1}(\&, N)$ are naturally isomorphic to the $K$-vectorspaces of derivations from the algebra to the bimodule modulo the inner derivations from the algebra to the bimodule.

(ii) $H^{0}(Q, M), H^{0}(\mathcal{L}, N)$ are naturally isomorphic to the sub- $K$ vector spaces of $M, N$ respectively that determine the inner derivation 0 -i.e. $H^{0}(Q, M)$ is naturally isomorphic to the $K$-vector-space generated by $\left\{m \in M \mid m_{R}-m_{L}=0\right\}$ and $H^{0}(\mathscr{L}, N)$ is naturally isomorphic to the $K$-vector-space generated by $\left\{n \in N \mid n_{R}=0\right\}$.

(iii) $H^{2}(Q, M), H^{2}(\&, N)$ are naturally isomorphic to the $K$-vectorspaces of equivalence classes of short singular extensions of $M$ by $Q$, $N$ by $\&$, respectively.

(iv) $H^{n}(a, M), H^{n}(\mathfrak{L}, N), n \geqq 3$, are naturally isomorphic to the $K$-vector-spaces of equivalence classes of singular extensions of length $n$ of $M$ by $\propto, N$ by $\&$, respectively.

We construct a cohomology theory for an arbitrary nonassociative algebra satisfying a set of identities $T$, within which the associative and Lie theories are special cases. Let $a$ be a $T$-algebra over the commutative ring with unit $K, M$ a $T$-bimodule for $Q$. We write $U(Q)$ for the universal multiplication algebra of $a$; that is, $U(a)$ is an associative algebra with unit such that all $a$-bimodules are right unital $U(a)$-modules in a natural fashion and conversely. For details of this, see Jacobson [4a] or Knopfmacher [5].

Following Gerstenhaber, we make the next two definitions.

Definition. $H^{2}(a, M)$ is the $K$-module of (not necessarily $K$-split) equivalence classes of short singular extensions of $M$ by $a$.

Definition. $H^{n}(Q, M), n \geqq 3$, is the $K$-module of (not necessarily $K$-split) equivalence classes of singular extensions of length $n$ of $M$ by $a$. 
Gerstenhaber [1] has shown that if $0 \rightarrow M^{\prime} \rightarrow M \rightarrow M^{\prime \prime} \rightarrow 0$ is an exact sequence of $a$-bimodules, then there are natural homomorphisms of $K$-modules $\delta^{n}$ so that the long sequence

$$
\begin{aligned}
0 & \rightarrow D\left(a, M^{\prime}\right) \rightarrow D(a, M) \rightarrow D\left(a, M^{\prime \prime}\right) \stackrel{\delta^{1}}{\rightarrow} H^{2}\left(a, M^{\prime}\right) \\
& \rightarrow H^{2}(a, M) \rightarrow H^{2}\left(a, M^{\prime \prime}\right) \stackrel{\delta^{2}}{\rightarrow} H^{3}\left(a, M^{\prime}\right) \rightarrow \cdots \\
& \rightarrow H^{n}\left(\left(, M^{\prime \prime}\right) \stackrel{\delta^{n}}{\rightarrow} H^{n+1}\left(a, M^{\prime}\right) \rightarrow \cdots\right.
\end{aligned}
$$

is exact, where $D(a$,$) is the functor which associates to each Q$ bimodule $M$ the $K$-module of derivations of $a$ to $M$.

Definition. An inner derivation functor is an epimorphism preserving subfunctor of $D(a$,$) , the derivation functor.$

Lemma. There is a 1-1 correspondence between inner derivation functors and left $U(a)$-submodules of $D(a, U(a))$.

With this lemma, we may speak of finitely generated inner derivation functors, meaning that the corresponding submodule of $D(a, U(a))$ is finitely $U(a)$ generated. Let $J(a, \quad)$ be a finitely generated inner derivation functor, generated by $\left\{d_{i}\right\}_{1}^{k}$.

Definition. $H_{J}^{1}(a, M)=D(Q, M) / J(Q, M)$.

Let $X_{i}, i=1, \cdots, k$, be the free $U(Q)$-module on one generator $x_{i}$, and regard $d_{i} \in D\left(Q, X_{i}\right)$. Let $Y \subseteq \oplus \sum_{1}^{k} X_{i}$ be the submodule generated by $\left\{a \sum_{1}^{k} d_{i} \mid a \in Q\right\}$. Let $C_{J}=\oplus \sum_{1}^{k} X_{i} / Y$.

Definition. $H_{J}{ }^{0}(a, M)=\operatorname{Hom}_{U(Q)}\left(C_{J}, M\right)$.

ThEOREM. If $0 \rightarrow M^{\prime} \rightarrow M \rightarrow M^{\prime \prime} \rightarrow 0$ is an exact sequence of $a$ bimodules, there are natural homomorphisms $\delta^{n}$ of $K$-modules so that the long sequence

$$
\begin{gathered}
0 \rightarrow H^{0}\left(a, M^{\prime}\right) \rightarrow H^{0}(a, M) \rightarrow H^{0}\left(a, M^{\prime \prime}\right) \stackrel{\delta^{0}}{\rightarrow} H^{1}\left(a, M^{\prime}\right) \\
\rightarrow \cdots \rightarrow H^{n}\left(a, M^{\prime \prime}\right) \stackrel{\delta^{n}}{\rightarrow} H^{n+1}\left(a, M^{\prime}\right) \cdots
\end{gathered}
$$

is exact.

In the classical associative and Lie theories, one takes for $J$ the inner derivation functor generated by those derivations which are in the Lie transformation algebra of the split null extension of $U(a)$ by $a$.

The above definitions and theorem can easily be modified to handle relative cohomology, unital cohomology, or cohomology of algebras and bimodules with involution. In the last case, we consider 
only derivations and morphisms commuting with involution. If $(a, \sigma)$ is a finite dimensional associative algebra with unit and involution over a field $K$ of characteristic $\neq 2,(M, \sigma)$ a unital bimodule with involution for $(a, \sigma)$, then, by an easy extension of a theorem of Harris [2]

THEOREM. $H^{n}((Q, \sigma),(M, \sigma)) \simeq \operatorname{Ext}^{n}{ }_{(U(Q), \bar{\sigma})}((Q,-\sigma),(M, \sigma))$, where $(U(Q), \bar{\sigma})$ has the property that any unital bimodule with involution for $(Q, \sigma)$ is a right associative unital module for $(U(Q), \bar{\sigma})$.

For $n \geqq 4, K$ a field of characteristic $\not 2,(a, \sigma)$ as above, we consider the Jordan matrix algebra $\mathfrak{H}_{n}(a, R)$, where $R$ is any canonical involution. $(Q,-\sigma)$ is a bimodule with involution for $(a, \sigma)$ and so determines a Jordan bimodule $C$ for $\mathfrak{F C}_{n}(a, R)$.

THEOREM. There is an inner derivation functor $J\left(\mathcal{F}_{n}(Q, R)\right.$, ) such that $\left.H_{J}^{k}\left(\mathcal{F C}_{n}(Q, R), M\right) \simeq \operatorname{Ext}_{U(\mathcal{H}}^{k} \mathcal{C}_{n}(Q, R)\right)(C, M)$ for all $k \geqq 0$ and all $\mathcal{F}_{n}(Q, R)$-bimodules $M$.

CoRollary. $\mathcal{F C}_{n}(Q, R)$ is separable if and only if $C$ is a projective $\mathfrak{F C}_{n}(Q, R)$ bimodule.

CoRollary. $\mathfrak{H C}_{n}(a, R)$ is separable if and only if for all $\mathfrak{K C}_{n}(a, R)$ bimodules $M, D\left(\mathfrak{F C}_{n}(Q, R), M\right)=J\left(\mathfrak{F C}_{n}(Q, R), M\right)$.

Definition. $I\left(\mathcal{H C}_{n}(Q, R), \quad\right)$ is the inner derivation functor such that $I\left(\mathcal{H C}_{n}(a, R), M\right)=\left\{\sum\left[R_{a} R_{m}\right] \mid\{a\} \subseteq \mathcal{H C}_{n}(a, R),\{m\} \subseteq M\right\}$.

Corollary. If characteristic $K \times n$, then $\mathfrak{F C}_{n}(Q, R)$ is separable if and only if for all $\mathcal{H C}_{n}(Q, R)$ bimodules $M, D\left(\mathcal{C}_{n}(Q, R), M\right)$ $=I\left(\mathcal{H C}_{n}(\AA, R), M\right)$.

We can extend the obstruction theory of Hochschild [3] for associative algebras to associative algebras with involution and to Jordan matrix algebras over them. If $(\alpha, \sigma),(B, \sigma)$ are associative with

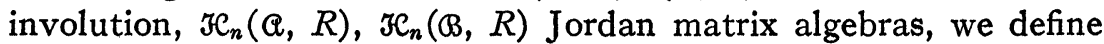
"outer multiplication" algebras and notions of "representations" of $(Q, \sigma)$ into $(B, \sigma)$ and $\mathfrak{H}_{n}(Q, R)$ into $\mathfrak{H}_{n}(B, R)$ in a manner similar to that of Hochschild.

THEOREM. There is an $(a, \sigma)$-bimodule $(N, \sigma)$ satisfying:

(i) To each representation $\phi:(a, \sigma) \rightarrow(B, \sigma)$, there corresponds an element $\tau(\phi) \in H^{3}((a, \sigma),(N, \sigma))$ and the map $\phi \rightarrow \tau(\phi)$ is an epimorphism.

(ii) $\phi$ is derived from a sequence $0 \rightarrow(B, \sigma) \rightarrow(\mathcal{E}, \sigma) \rightarrow(Q, \sigma) \rightarrow 0$ (see Hochschild) if and only if $\tau(\phi)=0$. 
(iii) If $\tau(\phi)=0$, the $K$-vector-space of equivalence classes of sequences in (ii) yielding $\phi$ is isomorphic to $H^{2}((\propto, \sigma),(N, \sigma))$.

THEOREM. There is an $\mathcal{H}_{n}(\mathfrak{Q}, R)$-bimodule $M$ satisfying:

(i) To each representation $\phi: \mathcal{H C}_{n}(Q, R) \rightarrow \mathcal{H}_{n}(\Theta, R)$ there corresponds an element $\tau(\phi) \in H^{3}\left(\mathcal{H C}_{n}(\alpha, R), M\right)$ and the map $\phi \rightarrow \tau(\phi)$ is an epimorphism.

(ii) $\phi$ is derived from a sequence $0 \rightarrow \mathcal{H C}_{n}(B, R) \rightarrow \mathcal{H C}_{n}(\mathcal{E}, R) \rightarrow \mathcal{H C}_{n}(Q, R)$ $\rightarrow 0$ if and only if $\tau(\phi)=0$.

(iii) If $\tau(\phi)=0$, the $K$-vector-space of equivalence classes of sequences in (ii) yielding $\phi$ is isomorphic to $H^{2}\left(\mathcal{K}_{n}(\propto, R), M\right)$.

If $\mathfrak{g}$ is a Jordan algebra over a field $K$ of characteristic $\neq 2, M$ a Jordan bimodule for $g$, Koecher [6] has defined a Lie algebra with involution $\left(K_{s}(\mathcal{\jmath}), \epsilon\right)$ on the vector space $\mathfrak{g} \oplus \overline{\mathfrak{g}} \oplus R(\mathfrak{g}) \oplus[R(\mathfrak{g}) R(\mathcal{g})]$, where $\bar{g}$ is linearly isomorphic to $g$, and a Lie bimodule with involution $\left(i_{s}(M), \epsilon\right)$ for $\left(K_{s}(g), \epsilon\right)$. Let $I\left(K_{s}(g), \epsilon\right)$ be the classical inner derivation previously discussed.

Theorem. $D(g, M) / I(g, M) \simeq D\left(K_{s}(g), i_{s}(M)\right) / I\left(K_{s}(g), i_{s}(M)\right)$ $\simeq D\left(\left(K_{s}(\mathcal{J}), \epsilon\right),\left(i_{s}(M), \epsilon\right)\right) / I\left(\left(K_{s}(\mathcal{g}), \epsilon\right),\left(i_{s}(M), \epsilon\right)\right)$.

Koecher has also shown that $K_{s}(\mathfrak{g})$ is separable if and only if $g$ is separable. This yields a new proof (originally proved in Jacobson $[4 \mathrm{~b}])$ of

CoROLlARY. If $\mathrm{g}$ is a separable Jordan algebra over a field of characteristic 0 , then all derivations of $g$ into any $\mathrm{g}$-bimodule $M$ are of the form

$$
a \rightarrow a \sum\left[R_{b} R_{m}\right], \quad a \in \mathcal{J},\{b\} \subseteq \mathcal{J},\{m\} \subseteq M
$$

We define an algebra similar to Koecher's but on the vector space $\mathfrak{g} \oplus \overline{\mathfrak{J}} \oplus U(\mathfrak{g})^{-}$, where $U(\mathfrak{g})^{-}$is the Lie subalgebra of $U(\mathfrak{g})$ generated by the image of $\mathscr{g}$ under the canonical injection $\rho: \mathcal{J} \rightarrow U(\mathcal{g})$. We call this algebra $\left(K_{u}(\mathcal{\jmath}), \epsilon\right)$.

ThEOREM. There is an injection $i: H^{2}(g, M) \rightarrow H^{2}\left(\left(K_{u}(g), \epsilon\right)\right.$, $\left.\left(i_{s}(M), \epsilon\right)\right)$. The image of $i$ consists of those cohomology classes which are represented by cocycles vanishing on $\mathrm{g}^{\rho} \times \mathrm{g}^{\rho}$.

This implies the known

COROLLARY. If $g$ is a separable Jordan algebra over a field of characteristic $0, M$ a g-bimodule, then any extension of $M$ by $g$ splits. 


\section{BIBLIOGRAPHY}

1. M. Gerstenhaber, A uniform cohomology theory for algebras, Proc. Nat. Acad. Sci. USA 52 (1964), 626-629.

2. B. Harris, Derivations of Jordan algebras, Pacific J. Math. 9 (1959), 495-512.

3. G. Hochschild, Cohomology and representations of associative algebras, Duke Math. J. 14 (1947), 921-948.

4. N. Jacobson, (a) Jordan algebras (to appear);(b) General representation theory of Jordan algebras, Trans. Amer. Math. Soc. 70 (1951), 509-571.

5. J. Knopfmacher, Universal envelopes for non-associative algebras, Quart. J. Math. Oxford Ser. 13 (1962), 264-282.

6. M. Koecher, Embeddings of Jordan algebras in Lie algebras, Yale Notes, Yale University, New Haven, Conn., 1966.

YALE UNIVERsity 